\title{
Parameters variation effects on energy recovery for a freely floating wave energy converter
}

\author{
A. JABRALI ${ }^{1}$, R. KHATYR ${ }^{2}$, J. KHALID NACIRI ${ }^{3}$ \\ Laboratory of Mechanics, Faculty of Sciences Aïn Chock, B.P 5366, Maarif, Hassan II University, Casablanca, Morocco \\ Corresponding authors: E-Mail: $\left({ }^{1}\right)$ jabraliahmed10@gmail.com \\ (²) khatyrrabha@gmail.com \\ (3) naciriuh2c@gmail.com
}

\begin{abstract}
This study presents the effects of parameters variations on the recovered energy for a floating wave energy converter (WEC) device. The articulated multi body floating WEC under consideration consists of two cylinders connected by a flat plate. The connections between the parts of the WEC allow the rotational movements of cylinders and the plate. The aim of this paper is to investigate the coupled effect of the length of the plate with the amplitude and period of the wave on the recovered energy by the floating WEC. The results show that the value of the optimum length for the plate is related to the sea wave condition, and more particularly to the amplitude and wavelength of the oscillations of the free surface.
\end{abstract}

Keywords: Wave energy converter; wave characteristics; multi-body articulated system; parameters dimensioning.

\section{Introduction}

In recent decades, many wave energy converters technologies have been designed. Reviews of these technologies can be found in the references [1-2]. Among the many existing types of devices, the floating ones are of particular interest since they do not require costly and complex fixing systems for their exploitation. High performance floating energy recovery devices such as Wavebob [3], SEAREV [4] and AquaBuoy [5] systems are constantly improved by addressing basic factors such as power recovery systems (PTO), shapes used, and other parameters.

In the present paper, parameters sizing for a floating wave energy converter (WEC) device is studied. The considered floating WEC, a multi-body articulated system, consists of two cylinders connected with a flat plate. The connections between the parts of the WEC allow the rotational movements of cylinders and the plate and the entire system may perform translational movements. This study focuses on the case of two-dimensional movements of the WEC due to the action of waves that propagate perpendicular to the axis of the cylinders. The pressure and the viscous forces acting on the immerged surfaces of the cylinders are modeled by the Morison force equation [6], to which are added Archimedes and gravity forces.

The Newton laws written for the multi-body articulated system, whose movements have five degrees of freedom, result in a system of five nonlinear second-order differential equations governing the motion of the WEC which is solved numerically by a fourth order Runge-Kutta method [7]. The results show the effects of the length of the relating plate on the efficiency of the wave energy converter and illustrate that varying this parameter has a significant effect on the energy recovery.

\section{Mathematical Formulation}

Let us consider the non-inertial reference frame $\mathcal{R}(0, \vec{x}, \vec{y}, \vec{z})$, where 0 is an arbitrary point taken at the moving free surface of the fluid and $\vec{y}$ is the upward vertical. The considered WEC consists of two cylinders, of centers $\mathrm{O}_{1}$ and $\mathrm{O}_{2}$, and radius $R_{1}$ and $R_{2}$ respectively, connected by a flat plate of center $\mathrm{G}$ and length $\mathrm{L}$.

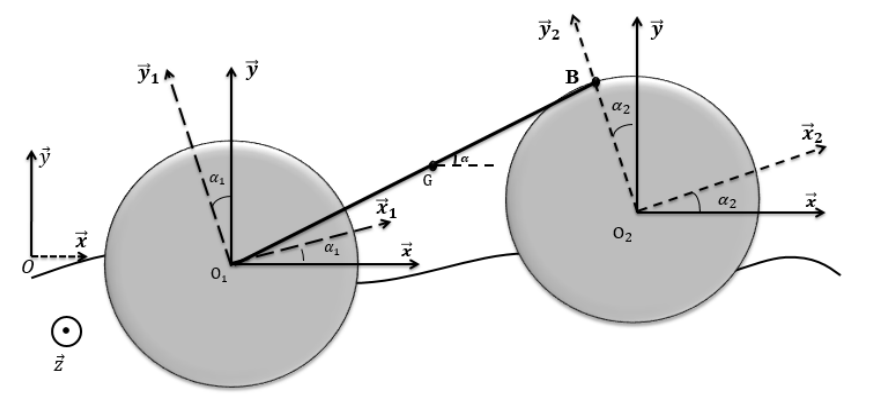

Bottom

Fig. 1. Schema of the floating WEC

Taking into account the connections between parts of the system as shown in figure 1 , and since only plane movements of the WEC are considered, we introduce five degrees of freedom for the mechanical system which are the heave $\left(y_{1}\right)$, the surge $\left(x_{1}\right)$ and the pitch $\left(\alpha_{1}\right)$ for cylinder 1 , the pitch $\left(\alpha_{2}\right)$ for cylinder 2 and the angle $(\alpha)$ for the plate. Here $x_{1}, y_{1}$ are the Cartesian coordinate of $\mathrm{O}_{1}$ in the frame $\mathcal{R}(0, \vec{x}, \vec{y}, \vec{z}), \alpha_{1}$ (resp. $\left.\alpha_{2}\right)$ is the angle between $\vec{x}$ and $\overrightarrow{x_{1}}$ 
(resp. $\overrightarrow{x_{2}}$ ) where $\overrightarrow{x_{l}}(i=1,2)$ is the axis of the relative frame of reference $\mathcal{R}_{i}\left(\mathrm{O}_{\mathrm{i}}, \overrightarrow{x_{1}}, \overrightarrow{y_{l}}, \vec{z}\right)$ attached to cylinder $i$ and $\alpha$ is the angle between $\vec{x}$ and the plate $\mathrm{O}_{1} \mathrm{~B}$.

In the non-inertial frame $\mathcal{R}(0, \vec{x}, \vec{y}, \vec{z})$, Newton's second law of motion applied to each part of the system separately [cylinder 1 , cylinder 2, plate] is expressed for cylinder $i$ ( $i=1$ for cylinder 1 and $i=2$ for cylinder 2 ) as follows :

$$
\left[D_{i}\right]=\left[\tau_{p i}\right]+\left[\tau_{M i}\right]+\left[\tau_{A i}\right]+\left[\tau_{L o i}\right]+\left[\tau_{R i}\right]-\left[\tau_{e i}\right]
$$

where $\left[D_{i}\right]$ is the dynamic torsor, $\left[\tau_{p i}\right]$ represents the gravity force torsor, $\left[\tau_{M i}\right]$ is the Morison force torsor representing the inertia forces and viscous forces exerted by the fluid on the system, $\left[\tau_{A i}\right]$ is the Archimedes thrust torsor, $\left[\tau_{L o i}\right]$ represents the reactions torsor at connection between the cylinder $i$ and the plate, $\left[\tau_{R i}\right]$ is the forces torsor for the power take off system of the WEC and $\left[\tau_{e i}\right]$ is the inertia force torsor related to the non-inertial character of the considered reference frame.

For the plate, the Newton's second law of motion is written as :

$$
\left[D_{b}\right]=\left[\tau_{p b}\right]-\left[\tau_{L G_{1}}\right]-\left[\tau_{L G_{2}}\right]-\left[\tau_{e b}\right]
$$

where $\left[D_{b}\right]$ is the dynamic torsor, $\left[\tau_{p b}\right]$ represents the gravity force torsor, $\left[\tau_{L G_{1}}\right]$ represents the reactions torsor at the point $G$ between the cylinder 1 and the plate, $\left[\tau_{L G_{2}}\right]$ represents the reactions torsor at the point $G$ between the cylinder 2 and the plate, and $\left[\tau_{e b}\right]$ is the inertia force torsor related to the non-inertial character of the considered reference frame.

By inserting the expressions of the torsors in Eqs. (1) and (2), and after rearrangement, one obtains the following system of five coupled differential equations for the five degrees of freedom $x_{1}, y_{1}, \alpha, \alpha_{1}$ and $\alpha_{2}$ :

$$
\begin{aligned}
& M \ddot{x}_{1}-m^{\prime} L \sin \alpha \ddot{\alpha}-m^{\prime} L \cos \alpha \dot{\alpha}^{2}+m_{2} R_{2} \cos \alpha_{2} \ddot{\alpha}_{2}- \\
& m_{2} R_{2} \sin \alpha_{2} \dot{\alpha}_{2}^{2}+F_{m 1 x}+F_{m 2 x}-F_{\text {ar } 1 x}-F_{\text {ar } 2 x}=0 \\
& M \ddot{y}_{1}+m^{\prime} L \cos \alpha \ddot{\alpha}-m^{\prime} L \sin \alpha \dot{\alpha}^{2}+M g+ \\
& m_{2} R_{2} \sin \alpha_{2} \ddot{\alpha}_{2}+m_{2} R_{2} \cos \alpha_{2} \dot{\alpha}_{2}^{2}+F_{m 1 y}+F_{m 2 y}- \\
& F_{\text {ar } 1 y}-F_{a r 2 y}+M A_{m} \omega^{2} \cos (\omega t)=0
\end{aligned}
$$

$m^{\prime \prime} \sin \alpha \ddot{x}_{1}-m^{\prime \prime} \cos \alpha \ddot{y}_{1}+m^{\prime \prime \prime} L \ddot{\alpha}-m_{2} R_{2} \sin (\alpha-$ $\left.\alpha_{2}\right) \ddot{\alpha}_{2}+m_{2} R_{2} \cos \left(\alpha-\alpha_{2}\right) \dot{\alpha}_{2}-\left(F_{\text {ar } 1 x}-F_{\text {ar } 2 x}\right) \sin \alpha+$ $\left(F_{\text {ar1y }}-F_{\text {ar2y }}\right) \cos \alpha-m^{\prime \prime} \cos \alpha g=0$

$\ddot{\alpha}_{1}+\frac{2}{m_{1} R_{1}^{2}} \beta_{1}\left(\dot{\alpha}_{1}-\dot{\alpha}\right)=0$

$m_{2} R_{2} \cos \alpha_{2} \ddot{x}_{1}+m_{2} R_{2} \sin \alpha_{2} \ddot{y}_{1}-m_{2} R_{2} L \sin (\alpha-$

$\left.\alpha_{2}\right) \ddot{\alpha}-m_{2} R_{2} L \cos \left(\alpha-\alpha_{2}\right) \dot{\alpha}^{2}+\frac{3}{2} m_{2} R_{2}^{2} \ddot{\alpha}_{2}+$

$\beta_{2}\left(\dot{\alpha}_{2}-\dot{\alpha}\right)-R_{2} \cos \alpha_{2} F_{\text {ar } 2 x}-R_{2} \sin \alpha_{2} F_{\text {ar } 2 y}+$

$m_{2} R_{2} \sin \alpha_{2} g=0$ where $m_{i}$ is the mass of the cylinder $i, R_{\mathrm{i}}$ and $\mathrm{L}_{\mathrm{i}}$ are respectively the radius and the length of the cylinder $i, m_{b}$ is the mass of the plate, $\mathrm{L}$ is the length of the plate, $\ddot{x}_{i}$ and $\ddot{y}_{\mathrm{i}}$ are the two accelerations of the cylinder $i$ along $\overrightarrow{0 x}$ and $\overrightarrow{0 y}$ axis respectively, $\ddot{x}_{G}$ and $\ddot{y}_{G}$ are the two accelerations of the plate along $\overrightarrow{\mathrm{Ox}}$ and $\overrightarrow{\mathrm{Oy}}$ axis respectively, $\ddot{\alpha}_{\mathrm{i}}$ is the angular acceleration of the cylinder $i, \ddot{\alpha}$ is the angular acceleration of the plate and $g$ represent gravity acceleration.

$F_{\text {mix }}$ and $F_{\text {miy }}$ are given by Morison equation respectively and are written as : $F_{\text {mix }}=\rho_{e} C_{m} V_{\mathrm{i}_{i}} \ddot{x}_{i}+\frac{1}{2} \rho_{e} C_{d} S_{i} \dot{x}_{i}\left|\dot{x}_{i}\right|$, $F_{\text {miy }}=\rho_{e} C_{m} V_{\mathrm{i}_{i}} \ddot{y}_{i}+\frac{1}{2} \rho_{e} C_{d} S_{i} \dot{y}_{i}\left|\dot{y}_{i}\right|$ where $\rho_{e}$ is the fluid density, $C_{m}$ is the added mass coefficient, $C_{d}$ is defined as drag coefficient, $S_{i}=R_{i} L_{i} \operatorname{Arccos}\left(\frac{y_{i}}{R}\right)$ is the wetted crosssection area of the cylinder $i$. The Archimedes forces $F_{\text {arix }}$ and $F_{\text {ariy }}$ along the $\overrightarrow{\mathrm{Ox}}$ and $\overrightarrow{\mathrm{Oy}}$ axis respectively, are defined by $-\rho_{\mathrm{e}} \mathrm{V}_{\mathrm{i}_{i}} g$, where $\mathrm{V}_{\mathrm{i}_{i}}$ is the immersed volume of cylinder $i$. The coefficient $\beta_{\mathrm{i}}$ is related to the power take off device, $\dot{\alpha}_{\mathrm{i}}$ is the angular velocity of the cylinder $i$ and $\dot{\alpha}$ is angular velocity of the plate.

The following notations where introduced $m^{\prime}=m_{2}+\frac{m_{b}}{2}$, $m^{\prime \prime}=m_{1}-m_{2}, m^{\prime \prime \prime}=m_{2}+\frac{m_{b}}{6}$ and $\mathrm{M}=m_{1}+m_{2}+m_{b}$ the total mass of the WEC. The expressions for Archimedes forces components are given in Appendix A. The system of coupled differential Eqs. (3)-(7) is numerically solved by using 4th order Runge-Kutta method.

\section{Results and discussion}

Figure 2 illustrates the incident wave elevation $\eta(t)=A_{m} \cos (\omega t)$ where $A_{m}$ is the wave amplitude of the wave, $\omega=\frac{2 \pi}{T}$ is the pulsation of the wave, $T$ is the wave period, here $A_{m}=0,08 m$ and $T=6 s$.

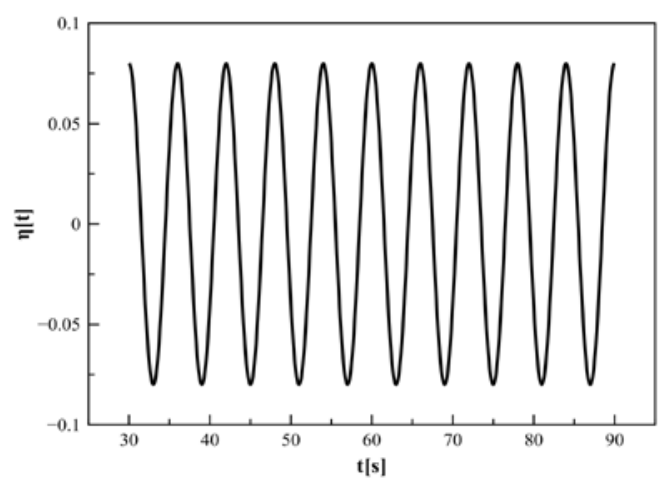

Fig. 2. Wave elevation as a function of the time

In order to find the optimum value of plate length a recovering coefficient, defined as the ratio of the recovered energy to the wave energy [8] during a period of time $t_{2}-t_{1}$, is introduced as follow:

$\xi=\frac{\mathrm{E}_{\mathrm{WEC}}}{\mathrm{E}_{\text {wave }}}=\frac{\int_{\mathrm{t}_{1}}^{\mathrm{t}_{2}} \beta_{\mathrm{i}}\left(\dot{\alpha}_{\mathrm{i}}(\mathrm{t})-\dot{\alpha}(\mathrm{t})\right)^{2} \mathrm{dt}}{L_{i} \cdot \rho_{e} g C A_{m}^{2}\left(t_{2}-t_{1}\right) / 2}$

where $L_{i}$ is the cylinder length, $C=\frac{\omega}{k}$ is the wave speed and $k$ is the wave number, here $k=0,11 m^{-1}$. 
Figure 3 represents the recovering coefficient obtained by the floating WEC versus the length of the plate, during $60 \mathrm{~s}$ for different waves where $R_{1}=R_{2}=0.0716, \quad L_{1}=$ $L_{2}=1.1, A_{\mathrm{m}}=0.08 \mathrm{~m}, \beta_{1}=0.29298 \mathrm{Ns} / \mathrm{m}, \beta_{2}=$ $0.66129 \mathrm{Ns} / \mathrm{m}, C_{d}=0.61$ and $C_{m}=7.46$.

This figure shows that the length of the plate and the wave period have an effect on the energy recovery. Moreover, when the length is $0,32 m$ for $T=2 s$ the device recovered a maximum energy of $6,77 \%$. However, for $L$ more than $0,36 \mathrm{~m}$, the recovered energy decreases.

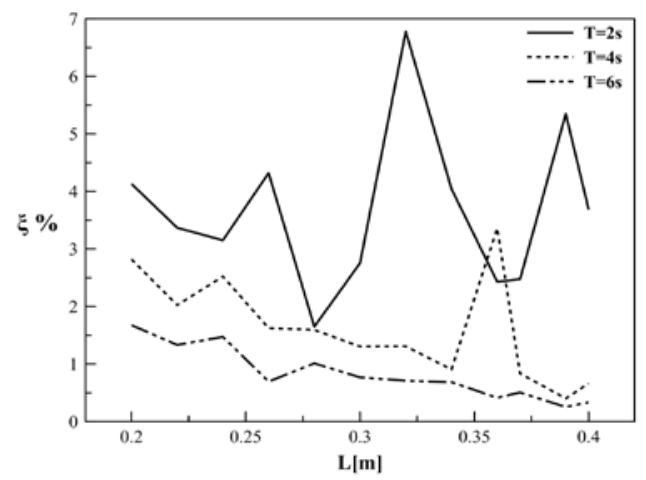

Fig. 3. The recovered coefficient as a function of the length of the plate for different wave periods

\section{Conclusion}

This paper has presented a test for dimensioning parameters for a floating wave energy converter in order to recover maximum energy. This test is based on varying one parameter where the others are fixed. The obtained results, shows that the recovered energy is larger in specific values of the length of the plate, and wave period. Furthermore, the optimum plate length is $0.32 \mathrm{~m}$ for $T=2 s$ and $A m=0.08 \mathrm{~m}$.

\section{References}

[1] J. Falnes, A review of wave-energy extraction, Marine structures, 20 (2007) 185-201.

[2] De O. Falcao AF., Wave energy utilization: a review of the technologies, Renewable and sustainable energy reviews, 14 (2009) 899-918.

[3] J. Weber, F. Mouwen, A. Parish, D. Robertson, Wavebob-research \& development network and tools in the context of systems engineering, Eighth European Wave and Tidal Energy Conference, Uppsala, Sweden, 2009, 416-420.

[4] J. Cordonnier, F. Gorintin, A. De Cagny, A.H., Clement, A. Babarit, SEAREV: Case study of the development of a wave energy converter, Renewable Energy, 80 (2015) 40-52.

[5] A. Weinstein, G. Fredrikson, M.J. Parks and K. Neislen, AquaBuOY-the offshore wave energy converter numerical modeling and optimization, Oceans'04, MTTS/IEEE techno-ocean'04, Kobe, Japan, (2004) 1854-1859.
[6] J.R. Morison, M.P. O’Brien, J.W Johnson and S.A. Schaaf, The forces exerted by surface waves on piles, Petroleum Transactions of AIME, 189 (1950) 149-157.

[7] W.H. Press, S.A. Teukolsky, W.T. Vetterling and B.P. Flannery, Numerical recipes in fortran 77, Cambridge University Press, 1992.

[8] J.N. Newman, Marine Hydrodynamics, M.I.T. Department of Naval Architecture and Marine Engineering, M.I.T Press, 1999.

\section{Appendix A. Archimedes Force}

The expression of the Archimedes force along the axis $\overrightarrow{0 x}$ is given by:

$$
\begin{aligned}
& F_{a r 1 x}=\rho_{e} g L_{1} R_{1} y_{1}\left(\sin \left(\theta_{L}+\frac{\eta_{1}}{R_{1}}\right)+\sin \left(\theta_{L}+\frac{\eta_{2}}{R_{1}}\right)\right)- \\
& \frac{\rho_{e} g L_{1} R_{1}^{2}}{4}\left(\cos \left(2\left(\theta_{L}+\frac{\eta_{1}}{R_{1}}\right)\right)-\cos \left(2\left(\theta_{L}+\frac{\eta_{2}}{R_{1}}\right)\right)\right) \\
& F_{a r 2 x}=\rho_{e} g L_{2} R_{2}\left(y_{1}+L \sin (\alpha)-\right. \\
& \left.R_{2} \cos \left(\alpha_{2}\right)\right)\left(\sin \left(\theta_{L 2}+\frac{\eta_{3}}{R_{2}}\right)+\sin \left(\theta_{L 2}+\frac{\eta_{4}}{R_{2}}\right)\right)- \\
& \frac{\rho_{e} g L_{2} R_{2}}{4}\left(\cos \left(2\left(\theta_{L 2}+\frac{\eta_{3}}{R_{2}}\right)\right)-\cos \left(2\left(\theta_{L 2}+\frac{\eta_{4}}{R_{2}}\right)\right)\right)
\end{aligned}
$$

The Archimedes force along the axis $\overrightarrow{\mathrm{Oy}}$ is written as:

$F_{\text {ar1 } y}=-\rho_{e} g L_{1} R_{1} y_{1}\left(\sin \left(\theta_{L}+\frac{\eta_{1}}{R_{1}}\right)+\sin \left(\theta_{L}+\frac{\eta_{2}}{R_{1}}\right)\right)+$

$\rho_{e} g L_{1} R_{1}^{2}\left(\theta_{L}+\frac{\eta_{1}+\eta_{2}}{2 R_{1}}+\frac{1}{4}\left(\sin \left(2\left(\theta_{L}+\frac{\eta_{1}}{R_{1}}\right)\right)+\right.\right.$

$\left.\left.\sin \left(2\left(\theta_{L}+\frac{\eta_{2}}{R_{1}}\right)\right)\right)\right)$

$F_{\text {ar2y }}=-\rho_{e} g L_{2} R_{2}\left(y_{1}+L \sin (\alpha)-\right.$

$\left.R_{2} \cos \left(\alpha_{2}\right)\right)\left(\sin \left(\theta_{L 2}+\frac{\eta_{3}}{R_{2}}\right)+\sin \left(\theta_{L 2}+\frac{\eta_{4}}{R_{2}}\right)\right)+$

$\rho_{e} g L_{2} R_{2}^{2}\left(\theta_{L 2}+\frac{\eta_{3}+\eta_{4}}{2 R_{2}}+\frac{1}{4}\left(\sin \left(2\left(\theta_{L 2}+\frac{\eta_{3}}{R_{2}}\right)\right)+\right.\right.$

$\left.\left.\sin \left(2\left(\theta_{L 2}+\frac{\eta_{4}}{R_{2}}\right)\right)\right)\right)$

with:

$$
\begin{aligned}
& \theta_{L}=\operatorname{arcos}\left(\frac{y_{1}}{R_{1}}\right), \theta_{L 2}=\operatorname{arcos}\left(\frac{y_{1}+L \sin (\alpha)-R_{2} \cos \left(\alpha_{2}\right)}{R_{2}}\right), \\
& \eta_{1}=A_{m} \cos \left(\omega t-k\left(x_{1}-R_{1} \sin \theta_{L}\right)\right) \\
& \eta_{2}=A_{m} \cos \left(\omega t-k\left(x_{1}+R_{1} \sin \theta_{L}\right)\right) \\
& \eta_{3}=A_{m} \cos \left(\omega t-k\left(x_{1}+L \cos \alpha+R_{2} \sin \alpha_{2}-R_{2} \sin \theta_{L 2}\right)\right) \\
& \eta_{4}=A_{m} \cos \left(\omega t-k\left(x_{1}+L \cos \alpha+R_{2} \sin \alpha_{2}+R_{2} \sin \theta_{L 2}\right)\right)
\end{aligned}
$$

